\title{
The PTPN22 allele encoding an R620W variant interferes with the removal of developing autoreactive B cells in humans
}

\author{
Laurence Menard, ${ }^{1}$ David Saadoun, ${ }^{1}$ Isabelle Isnardi, ${ }^{1}$ Yen-Shing Ng, ${ }^{1}$ Greta Meyers, ${ }^{1}$ \\ Christopher Massad, ${ }^{1}$ Christina Price, ${ }^{1}$ Clara Abraham, ${ }^{2}$ Roja Motaghedi, ${ }^{3}$ \\ Jane H. Buckner, ${ }^{4}$ Peter K. Gregersen, ${ }^{5}$ and Eric Meffre ${ }^{1}$
}

\begin{abstract}
1Department of Immunobiology and 2Department of Medicine, Section of Digestive Diseases, Yale University School of Medicine, New Haven Connecticut, USA. ${ }^{3}$ Department of Pediatrics, Weill Medical College of Cornell University, New York, New York, USA. ${ }^{4}$ Translational Research Program, Benaroya Research Institute, Seattle, Washington, USA. ${ }^{5}$ Robert S. Boas Center for Genomics and Human Genetics, The Feinstein Institute for Medical Research, Manhasset, New York, USA.
\end{abstract}

\begin{abstract}
Protein tyrosine phosphatase nonreceptor type 22 (PTPN22) gene polymorphisms are associated with many autoimmune diseases. The major risk allele encodes an $\mathrm{R} 620 \mathrm{~W}$ amino acid change that alters $\mathrm{B}$ cell receptor (BCR) signaling involved in the regulation of central B cell tolerance. To assess whether this PTPN22 risk allele affects the removal of developing autoreactive $B$ cells, we tested by ELISA the reactivity of recombinant antibodies isolated from single B cells from asymptomatic healthy individuals carrying one or two PTPN22 risk allele(s) encoding the PTPN22 R620W variant. We found that new emigrant/transitional and mature naive B cells from carriers of this PTPN22 risk allele contained high frequencies of autoreactive clones compared with those from non-carriers, revealing defective central and peripheral B cell tolerance checkpoints. Hence, a single PTPN22 risk allele has a dominant effect on altering autoreactive $B$ cell counterselection before any onset of autoimmunity. In addition, gene array experiments analyzing mature naive B cells displaying PTPN22 risk allele(s) revealed that the association strength of PTPN22 for autoimmunity may be due not only to the impaired removal of autoreactive B cells but also to the upregulation of genes such as CD40, TRAF1, and IRF5, which encode proteins that promote $B$ cell activation and have been identified as susceptibility genes associated with autoimmune diseases. These data demonstrate that early $B$ cell tolerance defects in autoimmunity can result from specific polymorphisms and precede the onset of disease.
\end{abstract}

\section{Introduction}

Autoimmune diseases affect about $5 \%$ of the population and are often characterized by the production of autoantibodies directed against self-antigens (1). An important role for B cells in autoimmune diseases is demonstrated by the successful treatment of patients with RA, type 1 diabetes (T1D), MS, and other autoimmune syndromes with anti-CD20 monoclonal antibodies that eliminate B cells (2-4). However, the underlying mechanisms that account for autoreactive $\mathrm{B}$ cells and autoantibody production in autoimmune diseases remain elusive.

We previously established in healthy donors that most developing autoreactive B cells are removed at two discrete steps. A central B cell tolerance checkpoint removes the vast majority of developing B cells that express polyreactive antibodies in the bone marrow (5). A peripheral B cell tolerance checkpoint further counterselects autoreactive new emigrant B cells before they enter the mature naive B cell compartment (5). In contrast, untreated active RA and SLE patients exhibit defective central and peripheral $\mathrm{B}$ cell tolerance checkpoints that result in the accumulation of self-reactive mature naive $B$ cells in their blood $(6,7)$. However, it is unclear whether these early B cell tolerance defects precede the onset of autoimmunity or result from

Authorship note: Laurence Menard and David Saadoun contributed equally to this work.

Conflict of interest: The authors have declared that no conflict of interest exists. Citation for this article: J Clin Invest. 2011;121(9):3635-3644. doi:10.1172/JCI45790. chronic ongoing inflammation processes. We recently reported that methotrexate or anti-TNF- $\alpha$ antiinflammatory treatments improved RA patients' condition without resetting early B cell tolerance checkpoints, suggesting that they may be controlled by intrinsic genetic factors (8).

Many susceptibility genes associated with autoimmune and inflammatory disease have recently been identified through genome-wide association studies (GWASs) (9). However, the mechanisms by which these gene variants contribute to the development of autoimmunity are unknown. We have focused on the role of a variant of the Protein tyrosine phosphatase nonreceptor type 22 (PTPN22) gene, which is associated with an increased risk for the development of many autoimmune diseases, including RA, T1D, and SLE (10-13). This variant is a single nucleotide change (cytidine to thymidine) at residue 1,858 that results in a single amino acid substitution from arginine to tryptophan at position 620 of the PTPN22/Lyp protein. Current data indicate that the 620W polymorphism in PTPN22/Lyp is a gain-of-function variant that leads to decreased $\mathrm{T}$ cell receptor and BCR signaling, which may in turn regulate the establishment of human $\mathrm{T}$ and $\mathrm{B}$ cell tolerance $(14,15)$. In this study, we have analyzed the impact of the PTPN22 risk allele on the establishment of B cell tolerance in healthy donors and found that it interferes with the removal of developing autoreactive $B$ cells. We thus demonstrate that early B cell tolerance defects common to RA, SLE, and T1D may result from specific polymorphisms and precede the onset of these autoimmune diseases. 
A

New emigrant B cells
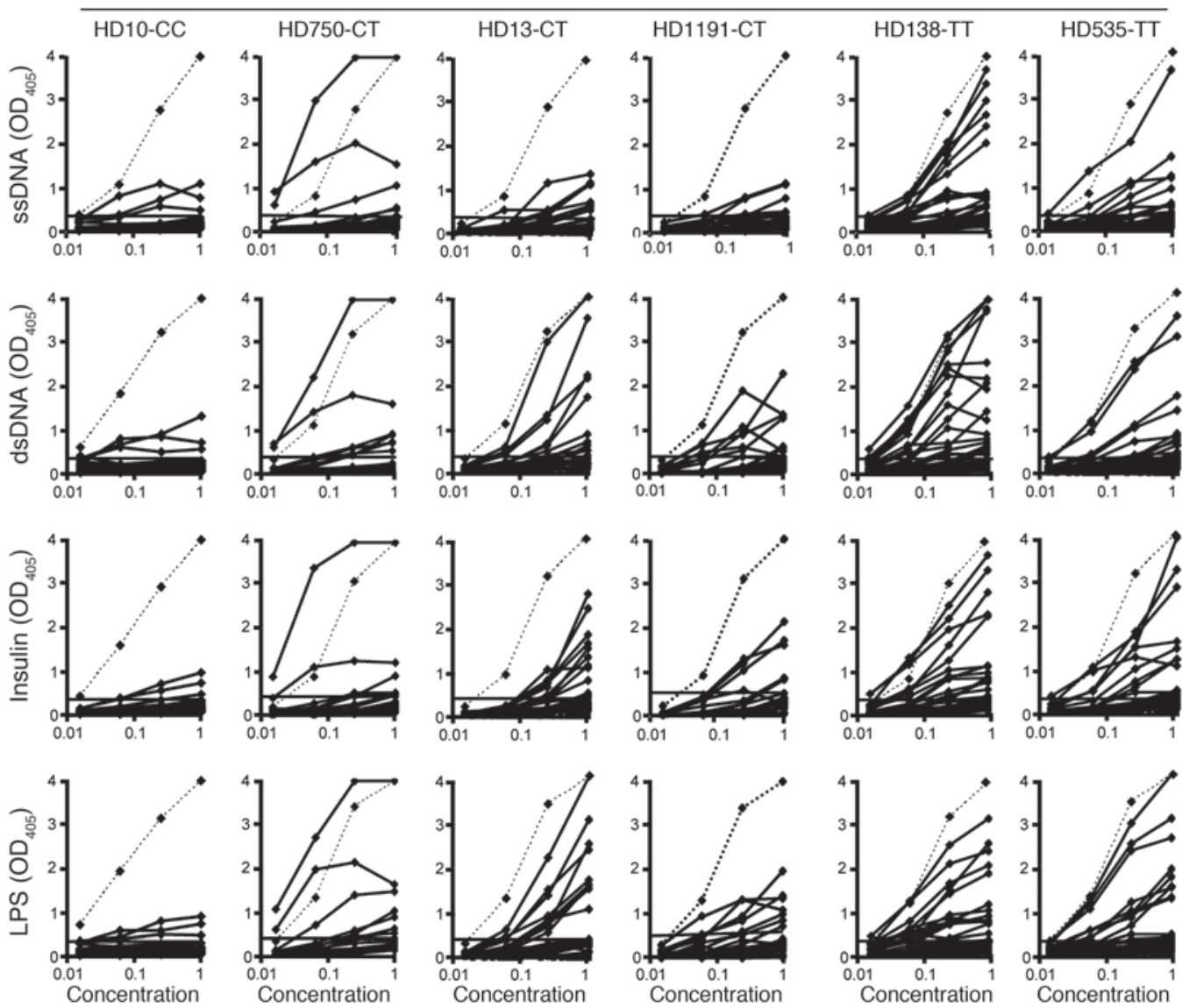

$(\mu \mathrm{g} / \mathrm{ml})$

$(\mu \mathrm{g} / \mathrm{ml})$

$(\mu \mathrm{g} / \mathrm{ml})$

$(\mu \mathrm{g} / \mathrm{ml})$

$(\mu \mathrm{g} / \mathrm{ml})$
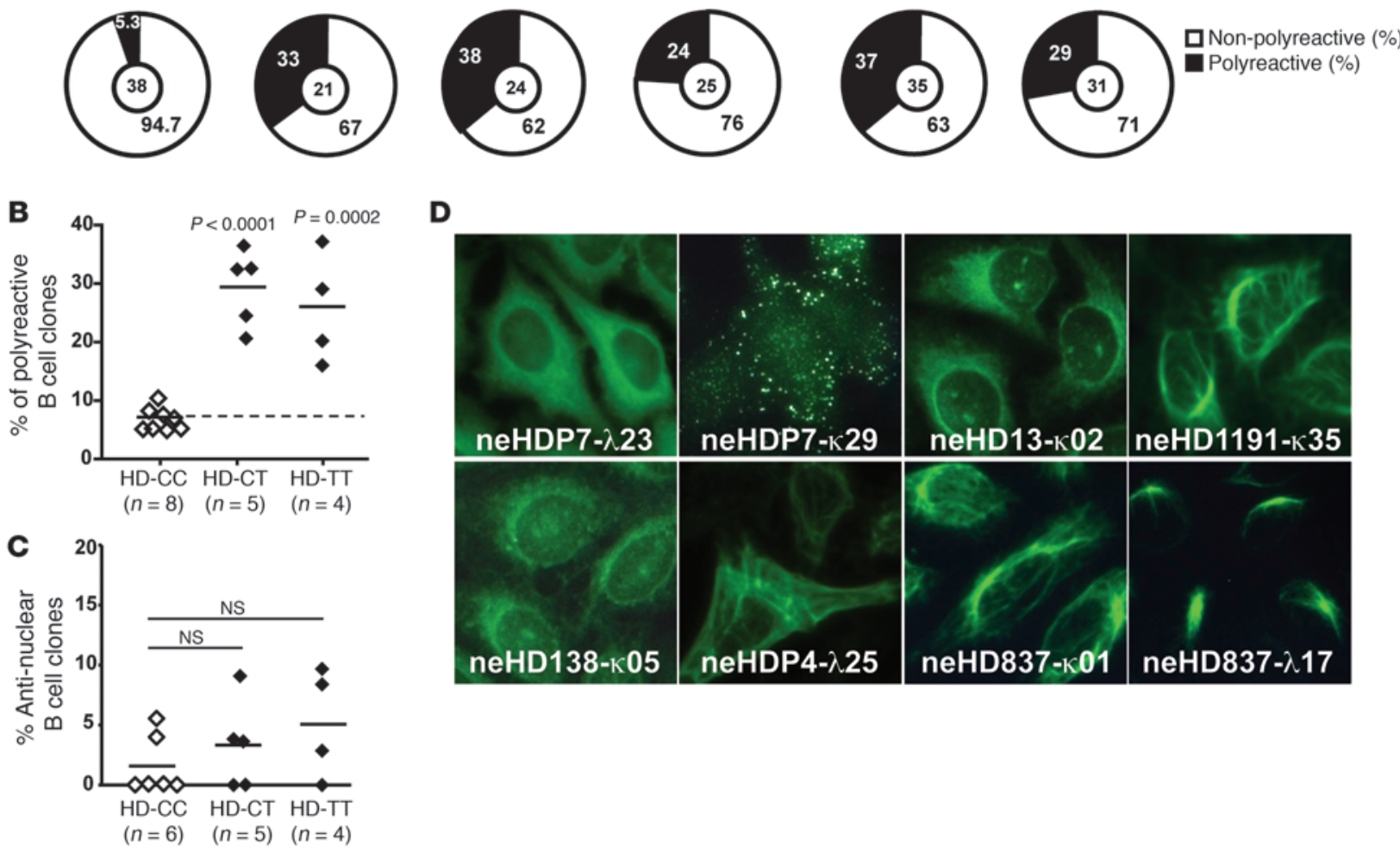
Figure 1

Altered central B cell tolerance checkpoint in healthy individuals carrying PTPN22 risk allele(s). (A) Antibodies from new emigrant/transitional B cells from healthy donors who did not carry the PTPN22 risk allele (HD-CC) or carried one (HD-CT) or two (HD-TT) PTPN22 risk allele(s) were tested by ELISA for reactivity against ssDNA, dsDNA, insulin and LPS. Polyreactive antibodies reacted against all 4 antigens. Dotted lines show ED38-positive control (5). Horizontal lines show cutoff $\mathrm{OD}_{405}$ for positive reactivity. For each individual, the frequency of polyreactive and nonpolyreactive clones is summarized in pie charts, with the number of antibodies tested indicated in the center. The frequencies of polyreactive (B) and anti-nuclear (C) new emigrant/transitional B cells are compared between healthy donors carrying or not carrying PTPN22 risk allele(s), and statistically significant differences are indicated. Each diamond represents an individual, and the horizontal bars show the average. (D) Autoreactive antibodies expressed by new emigrant (ne) B cells from PTPN22 risk allele carriers mostly show various cytoplasmic patterns of HEp-2 staining. Original magnification, $\times 40$.

\section{Results}

Impaired central B cell tolerance in bealthy donors carrying PTPN22 risk allele(s). The PTPN22 risk allele is associated with the development of autoimmune diseases such as RA and SLE, characterized by an impaired counterselection of developing autoreactive B cells $(6,7)$. To assess whether the central B cell tolerance checkpoint, which normally removes highly polyreactive and anti-nuclear developing B cells in the bone marrow, is affected by the presence of the PTPN22 risk allele(s), we cloned antibodies expressed by single

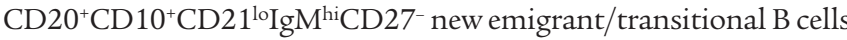
from 9 carrier healthy donors (Supplemental Tables 1-9) and tested their reactivity by ELISA (5). The reactivities of antibodies expressed by transitional/new emigrant B cells from healthy donors carrying one or two PTPN22 risk allele(s) were compared with those of their counterparts in non-carrier control donors (Figure 1 and refs. 5, $8,16-18)$. We found that polyreactive new emigrant/transitional B cells were significantly increased in all 5 healthy donors who carried one PTPN22 risk allele (T allele carriers; $21 \%-38 \%$ of the clones) compared with non-carrier healthy controls ( $\mathrm{C}$ allele individuals; 5\%-11\%) (refs. 5, 8, 16-18, Figure 1, A and B, and Supplemental Figure 1; supplemental material available online with this article; doi:10.1172/JCI45790DS1). Healthy donors who were homozygotes for the PTPN22 risk allele also displayed elevated frequencies of polyreactive clones in their transitional B cell compartment that

A

Mature naive $\mathrm{B}$ cells
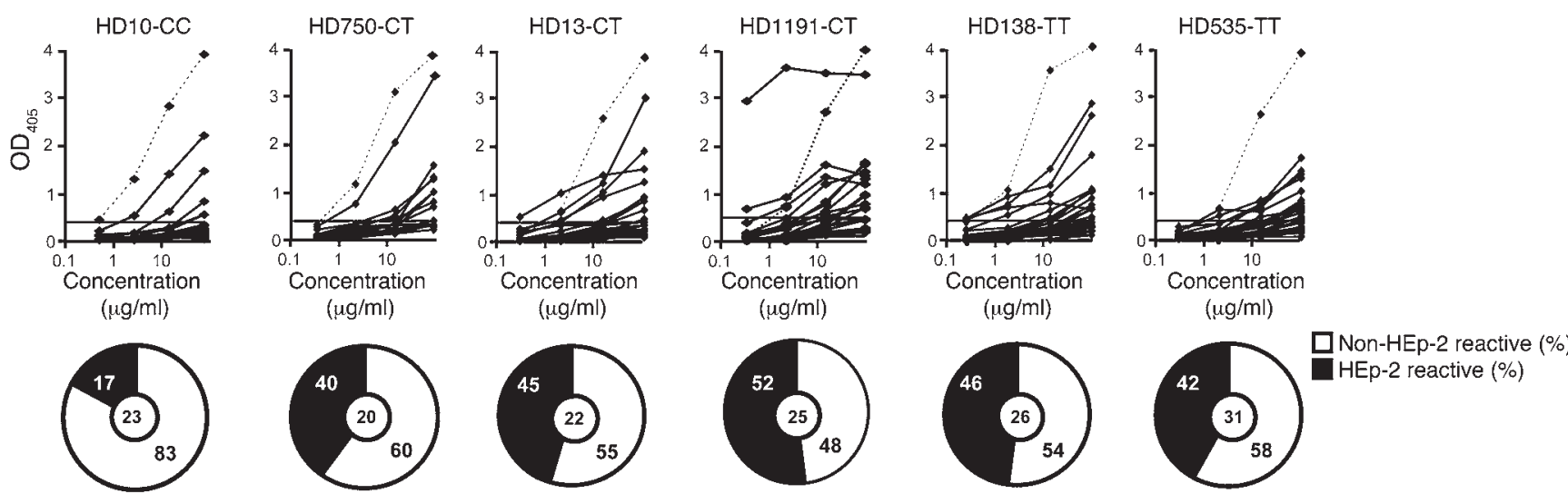

B

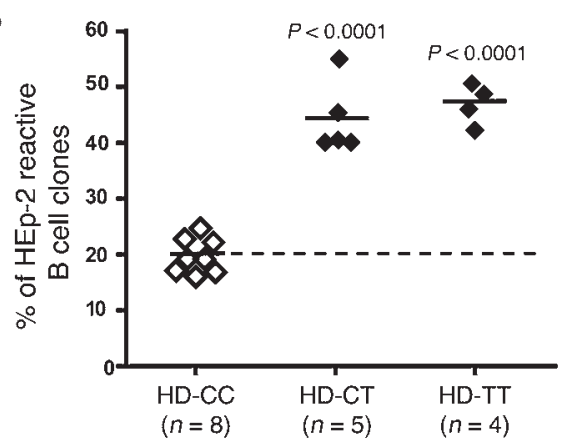

C

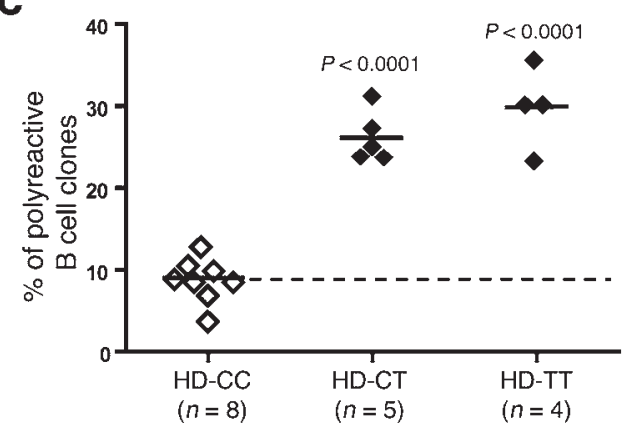

Figure 2

The PTPN22 risk allele interferes with the peripheral B cell tolerance checkpoint. (A) Antibodies from mature naive B cells from healthy donors who did not carry the PTPN22 risk allele (HD-CC) or carried one (HD-CT) or two (HD-TT) PTPN22 risk allele(s) were tested by ELISA for anti-HEp-2 cell reactivity. Dotted lines show ED38-positive control $(5,29)$. Horizontal lines show cutoff OD 405 for positive reactivity. For each individual, the frequency of HEp-2-reactive and nonreactive clones is summarized in pie charts, with the number of antibodies tested indicated in the center. Frequencies of HEp-2-reactive and polyreactive clones in the mature naive fraction of healthy donors carrying or not PTPN22 risk allele(s) are summarized in $\mathbf{B}$ and $\mathbf{C}$, respectively. 
New emigrant B cells
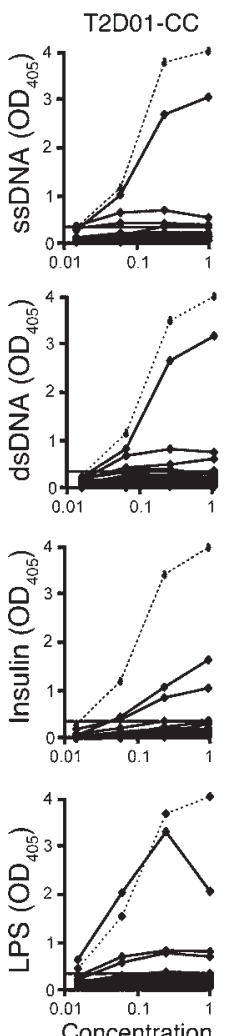

$(\mu \mathrm{g} / \mathrm{ml})$
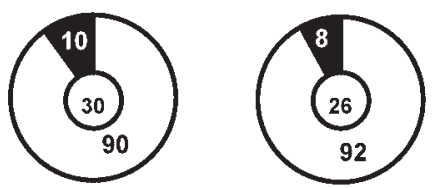
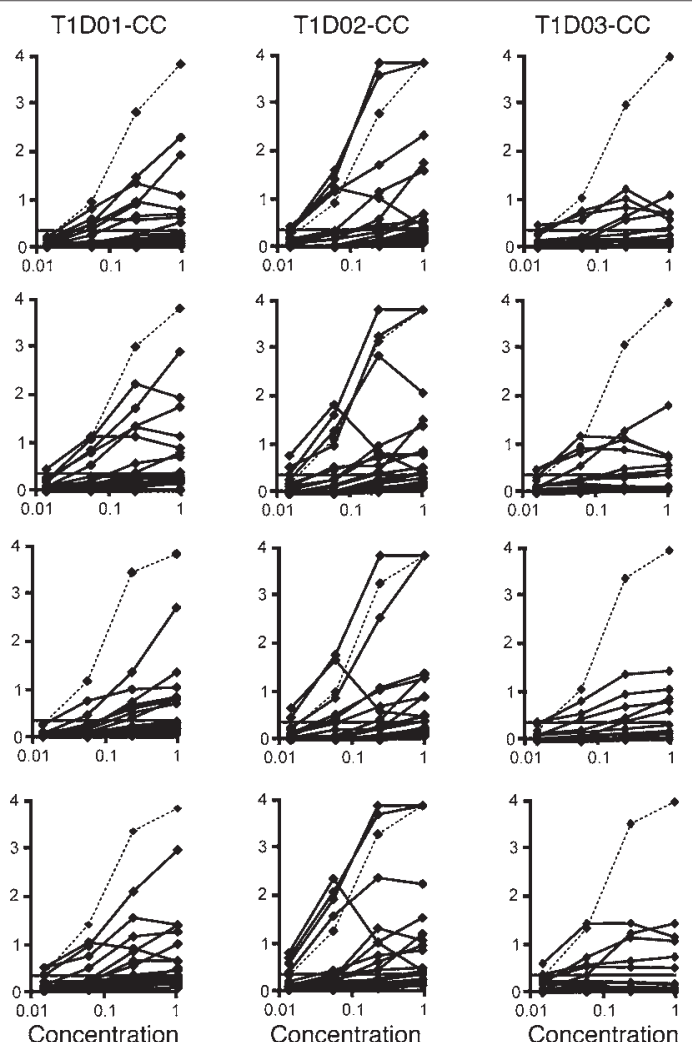

( $\mathrm{gg} / \mathrm{ml})$

$(\mu \mathrm{g} / \mathrm{ml})$
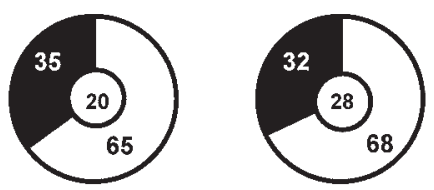
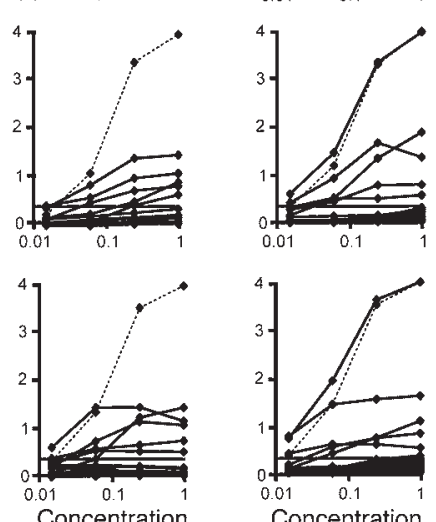

$(\mu \mathrm{g} / \mathrm{ml})$
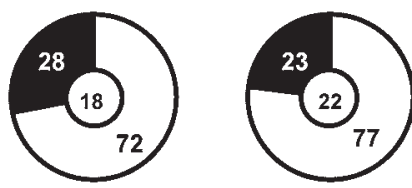
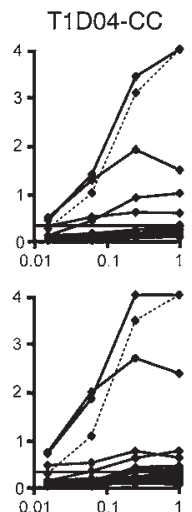

T1D05-CC
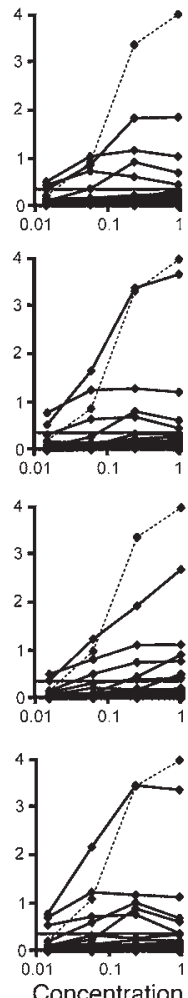

$(\mu \mathrm{g} / \mathrm{ml})$

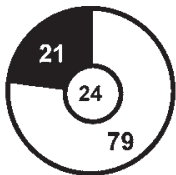

New emigrant $B$ cells
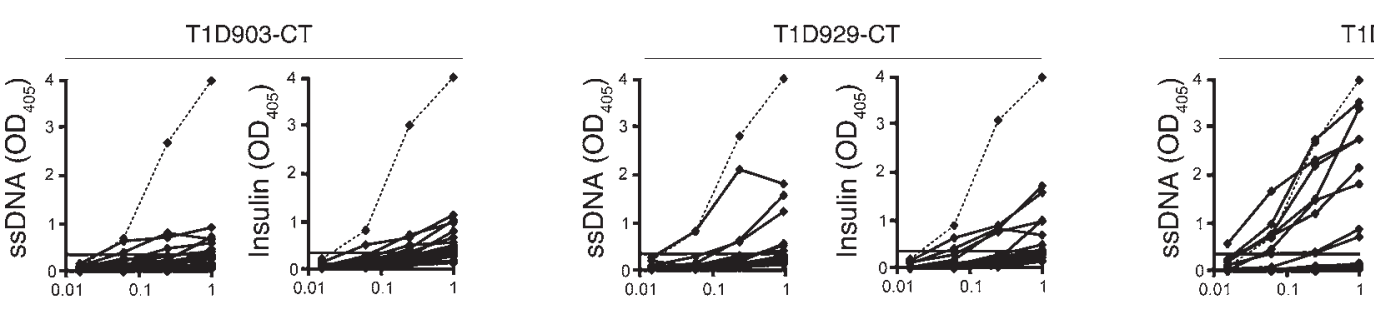

T1D340-TT

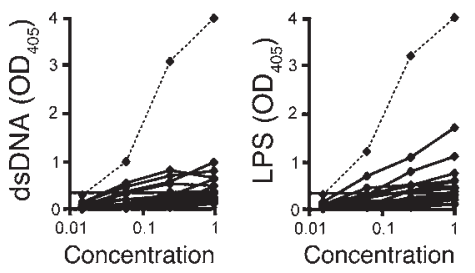

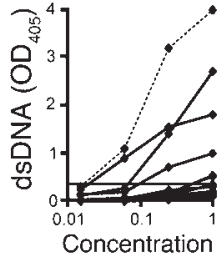

$(\mu \mathrm{g} / \mathrm{ml})$
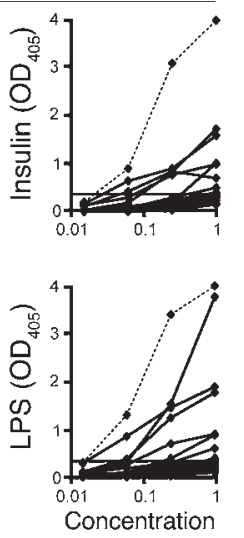

$(\mu \mathrm{g} / \mathrm{ml})$

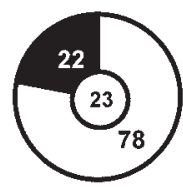

\begin{abstract}
$(\mu \mathrm{g} / \mathrm{ml})$
\end{abstract}

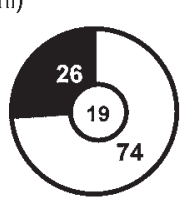

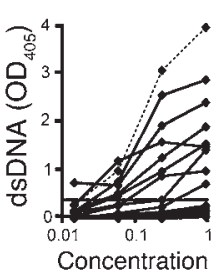

$(\mu \mathrm{g} / \mathrm{ml})$
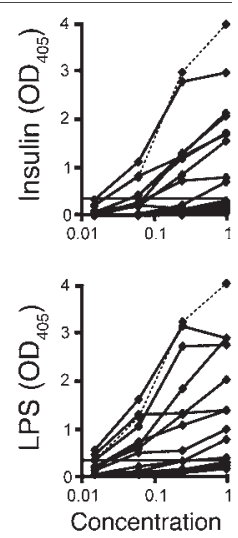

$(\mu \mathrm{g} / \mathrm{ml})$ 


\section{Figure 3}

Defective central B cell tolerance checkpoint in T1D patients. Antibodies from new emigrant B cells from non-carrier T2D and T1D (CC) and carrier T1D (CT or TT) patients were tested by ELISA for reactivity with ssDNA, dsDNA, insulin, and LPS. Dotted lines show ED38-positive control. Horizontal lines show cutoff $\mathrm{OD}_{405}$ for positive reactivity. For each individual, the frequency of polyreactive (black) and non polyreactive (white) clones is summarized in pie charts, with the number of antibodies tested indicated in the center.

were similar to those of heterozygote carriers, revealing a dominant effect of the PTPN22 risk allele on central B cell tolerance (Figure 1, $A$ and B). Using indirect immunofluorescence assays with HEp-2 cell-coated slides, we found that the proportion of anti-nuclear clones in new emigrant/transitional B cells from individuals carrying the PTPN22 risk allele(s) was modestly increased, but differences compared with non-carrier controls did not reach significance (Figure 1C). Self-reactive antibodies expressed by new emigrant/transitional B cells from heterozygote and homozygote PTPN22 risk allele carriers mostly recognized cytoplasmic structures including cytoskeleton components (Figure 1D). We conclude that the elevated frequency of polyreactive B cells in new emigrant/transitional B cells from healthy donors carrying one or two PTPN22 risk allele(s) demonstrates that central B cell tolerance is altered by the expression of overactive phosphatases encoded by the PTPN22 risk allele(s). The finding also reveals that the altered counterselection of developing autoreactive B cells previously found in patients with RA and SLE is likely to precede the onset of autoimmunity and is not a consequence or a by-product of chronic inflammatory conditions (6-8).

The PTPN22 risk allele also interferes with the peripheral B cell tolerance checkpoint. A second B cell tolerance checkpoint normally further eliminates autoreactive $\mathrm{B}$ cells that may recognize self-antigens in the periphery before they enter the $\mathrm{CD} 20^{+} \mathrm{CD} 10^{-} \mathrm{CD} 21^{+} \mathrm{IgM}{ }^{+} \mathrm{CD} 27^{-}$ mature naive $\mathrm{B}$ cell compartment (5). The impact of the PTPN22 risk allele on this peripheral B cell tolerance checkpoint was assessed by characterization of the reactivity of antibodies expressed by mature naive B cells from healthy donors carrying one or two PTPN22 risk allele(s) using an ELISA to screen for binding to antigens expressed by the HEp-2 cell line (Supplemental Tables 10-18) (5). The frequency of HEp-2-reactive mature naive B cells was significantly increased (40\%-55\%) in all individuals carrying either one or two PTPN22 risk alleles compared with non-carrier healthy donors (17\%-26\%) (Figure 2, A and B, and refs. 5, 8, 16-18). Mature naive B cells from PTPN22 risk allele carriers were also enriched in polyreactive clones compared with those from non-carrier control donors (Figure 2C). Thus, the presence of a single PTPN22 risk allele is sufficient to affect the removal of autoreactive $\mathrm{B}$ cells in the periphery and results in the accumulation of large numbers of mature naive $B$ cells expressing autoreactive antibodies.

$T 1 D$ patients suffer from defective early $B$ cell tolerance. The frequency of the PTPN22 risk allele is increased in T1D patients (12), yet the functionality of early B cell tolerance checkpoints has not been assessed in these subjects. Interestingly, anti-B cell therapies such as rituximab have shown some efficacy in delaying disease progression in T1D patients as well as in RA patients with defective early removal of developing autoreactive B cells $(2,4,6)$. To determine whether $B$ cell tolerance checkpoints are dysregulated in T1D, we analyzed the frequencies of autoreactive clones in the new emigrant/transitional and mature naive B cell compartments from T1D patients carrying or not the PTPN22 risk allele(s) and compared them with those in two non-autoimmune T2D patients (Figure 3, Figure 4, A and B, Supplemental Figures 2 and 3, and Supplemental Tables 19-34). Despite the fact that disease onset ranged widely, from 3 months to 28 years, all T1D patients showed an increased frequency of polyreactive clones in their new emigrant B cell compartments, demonstrating a defective central B cell tolerance checkpoint in T1D (Figure 3 and Figure 4A). Autoreactive new emigrant/transitional $B$ cell frequencies were similar to those in healthy donors carrying PTPN22 risk allele(s) (Figure 4A). In contrast, the frequencies of polyreactive antibodies expressed by new emigrant/transitional B cells in two non-autoimmune T2D patients who did not carry the PTPN22 risk allele were similar to those of their healthy donor counterparts, further demonstrating that an impaired central B cell tolerance was only associated with autoimmunity (Figure 3, Figure 4A, and Supplemental Tables 35 and 36). In addition, elevated frequencies of HEp-2-reactive and polyreactive $\mathrm{B}$ cells were also found in mature naive B cells from T1D but not T2D patients, suggesting a defective peripheral B cell tolerance checkpoint associated with T1D (Figure 4B, Supplemental Figures 2 and 3, and Supplemental Tables 37 and 38). Thus, T1D but not T2D patients suffer from defective central and peripheral B cell tolerance checkpoints similar to RA and SLE patients $(6,7)$, demonstrating that a failure to remove developing autoreactive B cells is common to all three autoimmune conditions and may favor the development of autoimmunity.

Similar autoreactive B cell frequencies were observed in T1D patients whether or not they harbored PTPN22 risk allele(s), suggesting that other polymorphisms may result in altered autoreactive B cell counterselection in these patients (Figure 4, A and B). This observation could also be extended to RA patients (Figure 4, C and D, and Supplemental Tables 39-46). Interestingly, many polymorphisms that have been reported to be associated with most of these autoimmune diseases, such as in $B L K, L Y N, B A N K 1$, and PTPN2 genes (9), which encode products mediating BCR signaling essential for the tuning of early B cell tolerance checkpoints, are therefore likely to also impact the removal of developing autoreactive B cells in humans.

The PTPN22 risk allele affects the expression of many other autoimmunity susceptibility genes. We next defined the impact of the PTPN22 risk allele on B cell physiology by performing gene array profiling and comparing transcripts expressed in mature naive B cells isolated from 8 healthy individuals carrying one or two PTPN22 risk allele(s) and 8 non-carrier control donors (Supplemental Table 47). Differentially expressed genes specifically associated with B cell biology are shown in Figure 5, Supplemental Figure 4, and Supplemental Tables 48 and 49. We found that mature naive B cells expressing PTPN22 risk allele(s) significantly upregulated the transcription of many genes belonging to three major $B$ cell activation pathways, i.e., the BCR, CD40, and TLR pathways converging to $\mathrm{NF}-\mathrm{\kappa B}$, and potentially counterbalancing the excessive signal dampening by the 620W PTPN22 risk allele (Figure 5 and Supplemental Table 48). Cytokine receptor transcripts, including $I L 4 R, I L 13 R, I L 17 R$, and IL21R, which stimulate B cell proliferation and differentiation, were found to be upregulated in B cells carrying PTPN22 risk allele(s) (Figure 5). Some HLA and polymerase genes were also found to be upregulated in mature naive $B$ cells carrying PTPN22 risk allele(s), suggesting an activated status of these cells (Supplemental Figure 4 and Supplemental Table 49). In addition, mature naive B cells from PTPN22 risk allele carriers differentially expressed many genes associated with many autoim- 
A
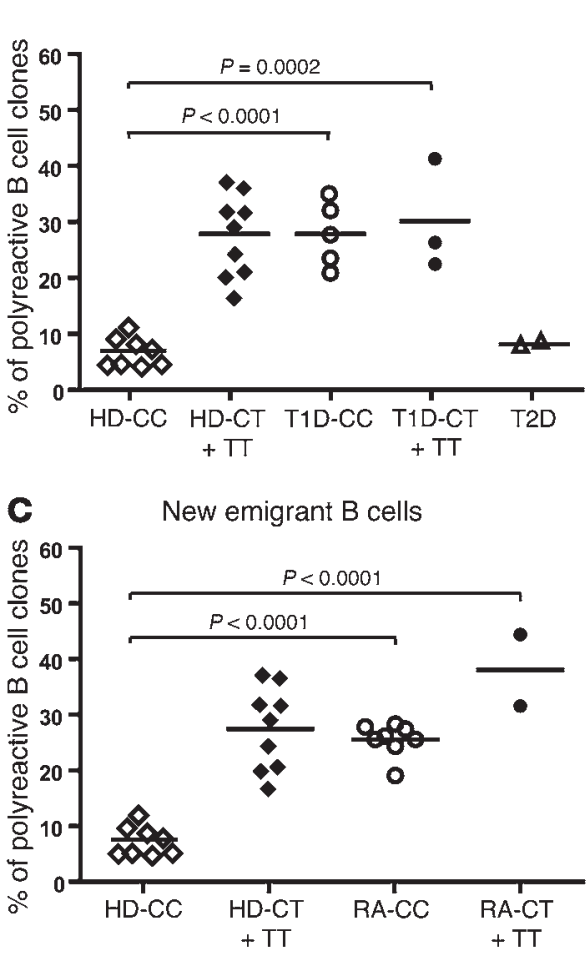

B

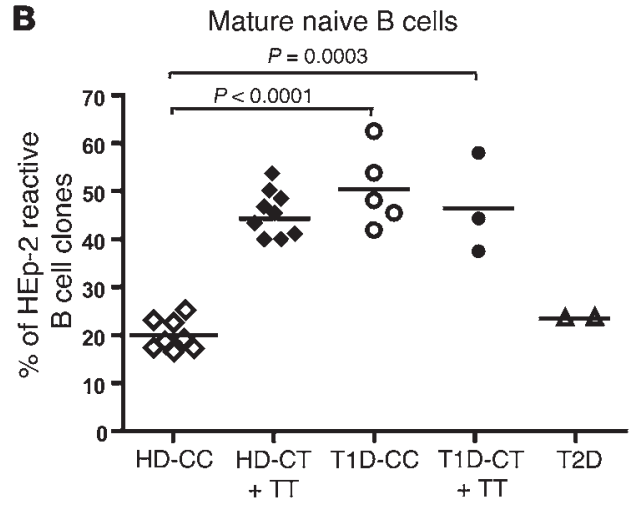

D

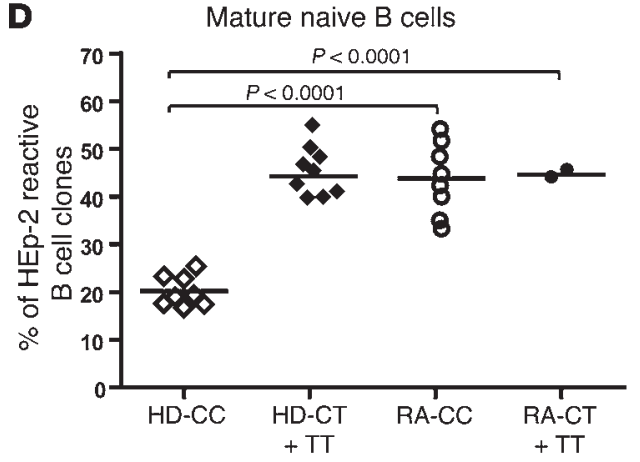

\section{Figure 4}

Healthy donors carrying the PTPN22 risk allele(s) display elevated frequencies of autoreactive $B$ cells similar to those in T1D and RA patients. The frequencies of polyreactive new emigrant/transitional (A) and HEp-2-reactive mature naive (B) B cells are compared between healthy donors and T1D patients carrying (T1D-CT+TT) or not (T1DCC) PTPN22 risk allele(s) as well as non-carrier T2D patients (T2D-CC). Similar analyses were performed in $\mathbf{C}$ and $\mathbf{D}$ with RA patients carrying (RA-CT+TT) or not (RA-CC) PTPN22 risk allele(s). Statistically significant differences are indicated. All T1D and RA patients display high frequencies of autoreactive clones similar to those from healthy donors carrying PTPN22 risk allele(s). mune diseases in mice or humans, including BLK, PTPN2, CD40, TRAF1, CD19, SLAM, and IRF5, as well as other genes belonging to the same pathways (Figure 5, Supplemental Table 48, and refs. 9, $19,20)$. To confirm the differential transcript regulation of several of these susceptibility and other pertinent genes, we utilized quantitative PCR to assess transcript levels in 16 risk allele carriers and 31 non-carrier control donors (Supplemental Table 50). Transcript level differences were validated for CD40, SLAMF6, CD19, IRF5, $B C L 2, T R A F 1, T R A F 2$, and MYD88 genes and reached significance (Figure 6 and Supplemental Figure 5). Transcript differences were almost significant for NFKB1 and RELB and were not validated for BLK, ICOSL, and DICER1 (Figure 6A and Supplemental Figure 5). An increase in CD40 and SLAMF6 but not CD19 on the surface of mature naive B cells from individuals carrying PTPN22 risk allele(s) was further validated by flow cytometry, and is likely to favor the activation of such B cells compared with those from non-carrier controls (Figure 6, B and C). In conclusion, the transcriptome of B cells harboring the PTPN22 risk allele was for the most part validated by quantitative PCR and flow cytometry and is characterized by the upregulation of many genes that have been found to be involved in the development of many autoimmune diseases and which encode molecules favoring B cell activation, proliferation, and survival.

Naive B cells carrying the PTPN22 risk allele are more responsive to CD40 stimulation. We further investigated the consequences of the increased cell surface expression of CD40 on naive B cells from PTPN22 risk allele carriers by stimulating in vitro these $B$ cells with increasing concentrations of soluble recombinant $\mathrm{CD} 40 \mathrm{~L}$ or $\mathrm{F}\left(\mathrm{ab}^{\prime}\right)_{2}$ anti-human IgM. We analyzed the expression of CD25, CD69, CD86, and FAS by flow cytometry after BCR or CD40 stimulation for 2 days. We found that naive B cells from PTPN22 risk allele carriers after CD40 triggering displayed signifi- cantly increased upregulation of CD69 and CD25 compared with B cells from PTPN22 non-carrier individuals (Figure 7, A and B). Enhanced responses to CD40L stimulation were also evident in B cells carrying the PTPN22 risk allele by stronger FAS/CD95 induction in these B cells than in B cells from non-carriers, whereas CD86 was induced similarly in both carrier and non-carrier B cells (Supplemental Figure 6). The enhanced naive B cell responses from PTPN22 risk allele carriers were specific to CD40 triggering because these B cells displayed CD69 and FAS/CD95 upregulation after BCR stimulation similar to that in non-carrier B cells (Figure 7 and Supplemental Figure 6). CD25 induction after BCR triggering seemed to be slightly enhanced on naive B cells harboring the PTPN22 risk allele, but differences compared with PTPN22 CC $B$ cells did not reach statistical significance (Figure 7). Thus, we conclude that the upregulation of CD40 and other molecules belonging to the CD40 signaling pathway observed in naive B cells from PTPN22 risk allele carriers correlates with enhanced B cell activation after CD40 stimulation.

\section{Discussion}

We demonstrated that the PTPN22 risk allele interferes with the removal of developing autoreactive B cells and allows the accumulation of large numbers of self-reactive mature naive $B$ cells in the periphery. The PTPN22 risk allele is associated with many autoimmune diseases, including RA, SLE, and T1D (10-13). Interestingly, T1D patients showed abnormal central and peripheral B cell tolerance checkpoints similar to those previously reported for RA and SLE patients, revealing that early B cell tolerance defects are common to all these autoimmune diseases and may favor autoimmunity. These impaired early B cell tolerance defects precede the onset of RA, SLE, and T1D because healthy individuals carrying the PTPN22 risk allele already display altered central 


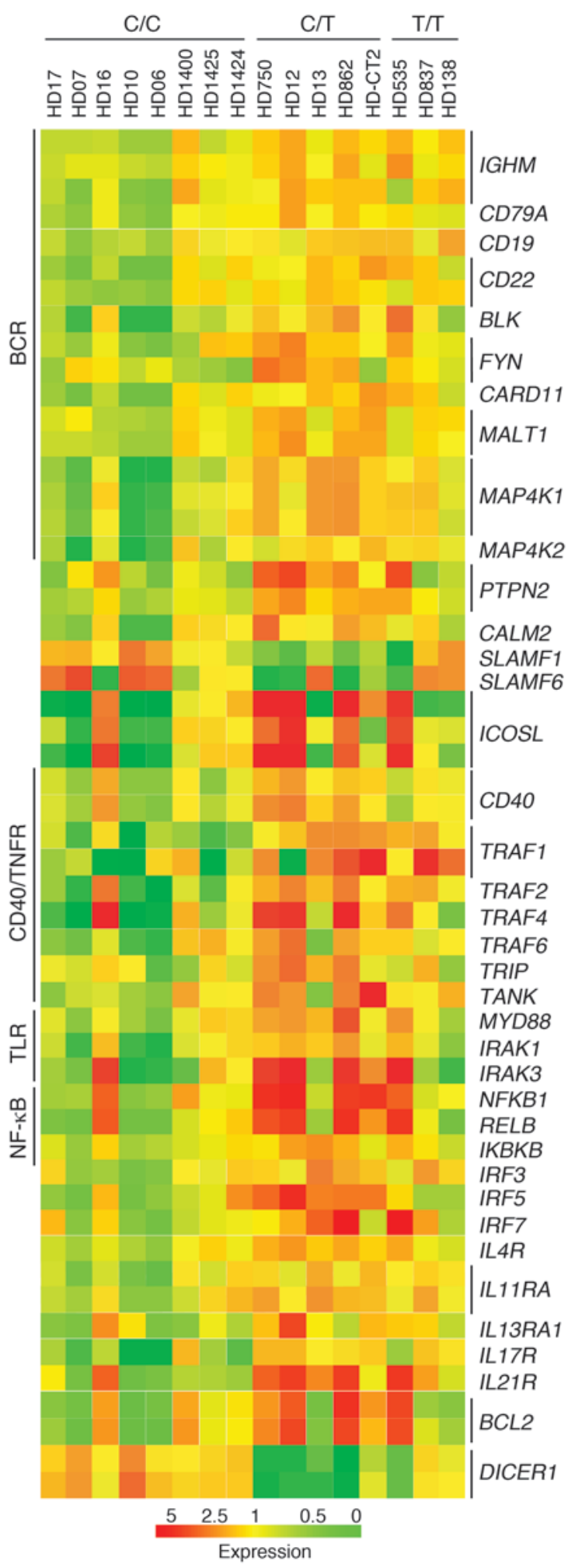

and peripheral tolerance checkpoints similar to those in patients with autoimmune diseases. Hence, this increased production of autoreactive naive B cells in PTPN22 risk allele carriers may play a role in the development of autoimmune syndromes by allowing the capture and the presentation of self-antigens to $\mathrm{T}$ cells, as previously postulated (21). The PTPN22 risk allele is likely to

\section{Figure 5}

Mature naive B cells from healthy donors carrying PTPN22 risk allele(s) upregulate the expression of many susceptibility genes associated with autoimmune diseases. Gene array comparisons of mature naive B cells from healthy donors (HD) carrying or not PTPN22 risk allele(s) using the Affymetrix Human Genome U133 Plus 2.0 Array. Selected transcripts differentially expressed and well known to be involved in B cell biology are presented. One or more probes are shown for each gene. Up- and downregulated transcripts are indicated in red and green, respectively. The magnitude of expression is depicted by the color bar. Additional differentially expressed genes are shown in Supplemental Figure 4.

directly interfere with the removal of developing autoreactive $\mathrm{B}$ cells by affecting BCR signaling. Indeed, the PTPN22 risk allele encodes a gain-of-function variant that leads to decreased BCR signaling, which has been shown to induce a defective central $\mathrm{B}$ cell tolerance checkpoint in humans (14-16). Hence, autoreactive immature B cells binding self-antigens may not generate proper BCR signaling in the presence the 620W PTPN22 phosphatases, resulting in a failure to induce $B$ cell tolerance mechanisms and the release of autoreactive $B$ cells in the periphery. In addition, the upregulation of $B C L 2$ transcription identified by gene array and quantitative PCR experiments in B cells expressing the PTPN22 risk allele may also interfere with the removal of autoreactive $\mathrm{B}$ cells as previously demonstrated in mice (22). Moreover, increased CD40 expression on naive B cells from PTPN22 risk allele carriers is also likely to favor developing B cell survival. It remains to be determined whether the upregulation of these survival and activating genes is a direct or indirect effect of the PTPN22 risk allele and potentially reflects compensatory mechanisms for the reduction in $\mathrm{BCR}$ signaling associated with the gain of function of 620W PTPN22 dephosphorylating enzymes.

Gene array experiments analyzing B cells from healthy individuals carrying PTPN22 risk allele(s) also revealed that the presence of 620W PTPN22 phosphatases affected the expression of genes such as PTPN2, CD40, TRAF1, SLAM, and IRF5 that have been found to be involved in the development of many autoimmune diseases ( 9 , 20). These genes encode molecules belonging to important pathways leading to $\mathrm{B}$ cell activation, including those initiated by the BCR, CD40, TLR, and cytokine receptors. The validation of gene upregulation by quantitative PCR and flow cytometry further demonstrates the importance of 620W PTPN22 phosphatases in $\mathrm{B}$ cell physiology, in that they favor B cell activation. Indeed, we demonstrated that the upregulated expression of CD40 on the cell surface of naive B cells from individuals carrying the PTPN22 risk allele correlated with a stronger $B$ cell activation after CD $40 \mathrm{~L}$ stimulation compared with B cells from non-carrier donors. The upregulated transcription of TRAF genes encoding components mediating CD40 functions, as well as IL-4R, IL-13R, and IL-21R, which play important roles in $\mathrm{B}$ cell proliferation and differentiation, is also likely to favor B cell activation in individuals carrying PTPN22 risk allele(s). Together, our results suggest that the increased frequency of autoreactive B cells combined with CD40linked hyperactive B cell features in PTPN22 risk allele carriers may favor self-antigen presentation and interactions with $\mathrm{T}$ cells, potentially leading to the development of autoimmunity. In line with this hypothesis is the increased IRF5 expression in B cells displaying PTPN22 risk allele(s). Indeed, an SLE risk haplotype is associated with elevated expression of IRF5 (23), and animal mod- 

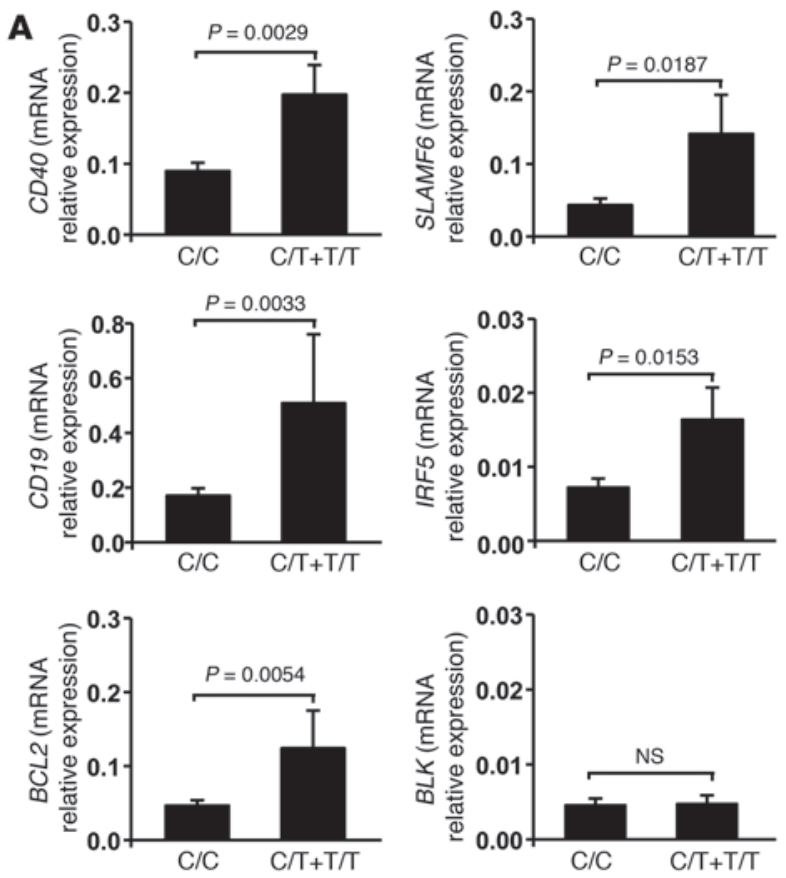
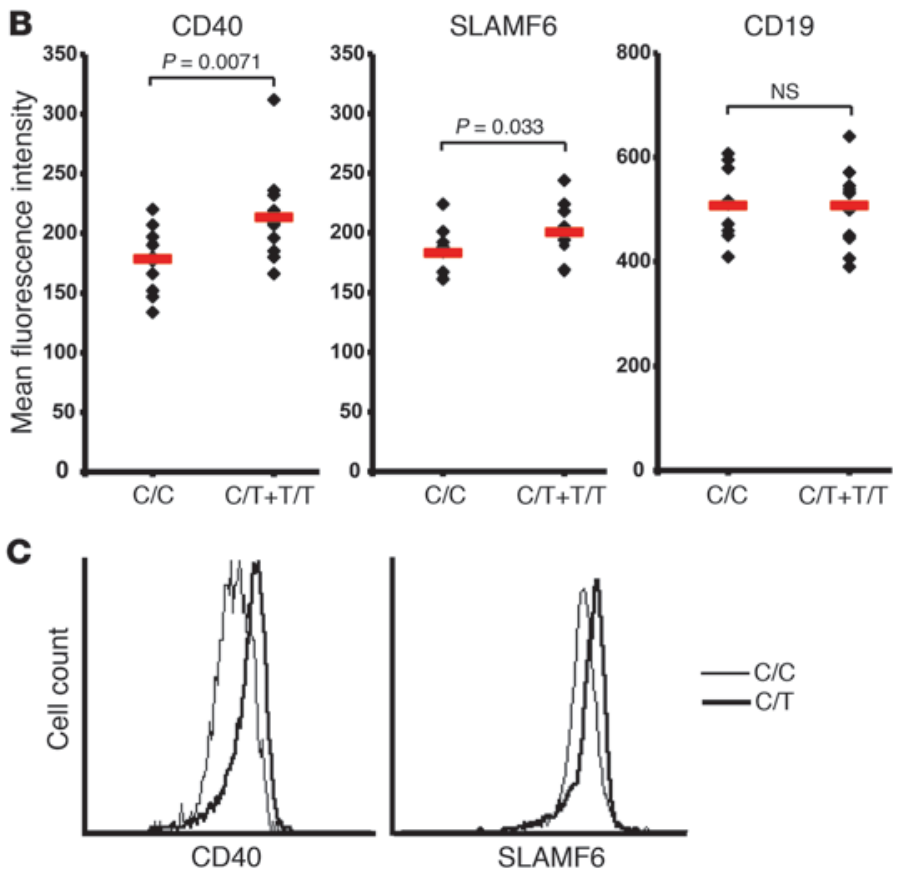

Figure 6

Mature naive B cells from healthy donors carrying PTPN22 risk allele(s) display a phenotype reflecting gene array profiling data. (A) Quantitative real-time PCR validates the increased transcription of many genes found upregulated in gene array profiling experiments. Gene expression was assessed by comparing 31 non-carrier healthy donors and 16 individuals carrying PTPN22 risk allele(s). Error bars represent the mean \pm SEM. (B) Flow cytometry analyses reveal the upregulation of CD40 and SLAMF6 but not CD19 on the surface of mature naive B cells from individuals carrying PTPN22 risk allele(s). Each diamond represents the mean fluorescence of the indicated molecules expressed on the surface of mature naive B cells from individuals carrying or not PTPN22 risk allele(s). Representative overlays for the indicated markers are shown in C.

els have also demonstrated that decreased IRF5 gene expression may thwart the development of autoimmunity (24). Thus, the strength of association of the PTPN22 risk allele with autoimmune disorders may be due not only to the altered BCR signaling resulting in defective early B cell tolerance checkpoints but also to the activation of potentially autoreactive B cells in the periphery.

Since the PTPN22 risk allele is also expressed in T cells and decreases $T$ cell receptor signaling (14), it is likely that $\mathrm{T}$ cell selection in the thymus and in particular the regulatory $\mathrm{T}$ cell repertoire will be also affected by the PTPN22 risk allele, thereby further contributing to the emergence of autoimmune diseases. However, in the absence of MHC alleles known to be associated with autoimmune diseases, it is unlikely that individuals carrying PTPN22 risk alleles will develop such conditions (9). The strong association of the fairly common PTPN22 risk allele among individuals of European descent with many humoral autoimmune diseases may represent an interesting target to thwart autoimmunity in a large pool of patients. Indeed, small molecules inhibiting PTPN22 enzyme activity may reset the threshold for counterselection of developing autoreactive B cells by increasing BCR signaling in individuals carrying PTPN22 risk allele(s). Interestingly, emerging literature also suggests that the PTPN22 risk allele may confer resistance to certain infections $(25,26)$. We postulate that the PTPN22 risk allele, especially common in Northern Europe (9), may in fact favor the production of better protective antibodies by allowing the development of polyreactive B cells. In line with this hypothesis, broadly neutralizing antibodies to HIV have been shown to be enriched in polyreactive clones $(27,28)$. Thus, pharmacological mimics of the
PTPN22 620W allele may also find utility in selected clinical situations to enhance the production of protective clones by the $\mathrm{B}$ cell arm of the immune system.

\section{Methods}

Patients and healthy donors. Healthy donors carrying or not the PTPN22 risk allele were enrolled through the Yale Autoimmunity Center of Excellence Core B (Supplemental Tables 47 and 50). Genotyping was assessed by TaqMan SNP genotyping (Applied Biosystems) or custom Sequenom iPlex array (Sequenom Inc.). Fresh, de-identified human blood was also collected from healthy control subjects following receipt of their consent by staff at the Feinstein Institute for Medical Research through the Institutional Review Board-approved Genes and Phenotype (GAP) Registry (IRB protocol 09-081), a national resource for genotype-phenotype studies (National Institute of Arthritis and Musculoskeletal and Skin Diseases project 1RC2AR059092). Additional frozen PBMC samples for this study were obtained from control and T1D participants in the Benaroya Research Institute Immune Mediated Disease Registry and Juvenile Diabetes Research Foundation (JDRF) Center for Translational Research. Research protocols were approved by the Benaroya Research Institute IRB. All T1D patients had absolute insulin deficiency and positive immunological markers of T1D and are described in Supplemental Table 51 with T2D patients. Patients RA01, RA02, RA03, RA04, RA05, RA06, RA11, and RA24 were previously analyzed, and none of those subjects carried the PTPN22 risk allele $(6,8)$. RA patient characteristics are reported in Supplemental Table 52. All samples were collected in accordance with IRB-approved protocols.

Cell staining and sorting. Peripheral B cells were purified from the blood of patients and control donors by positive selection using CD20 magnetic 
A

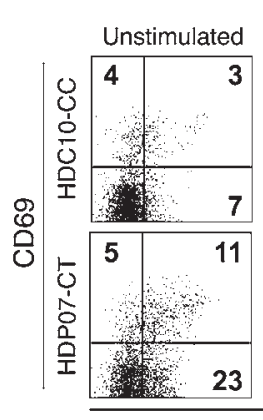

$\mathrm{CD} 40 \mathrm{~L}$

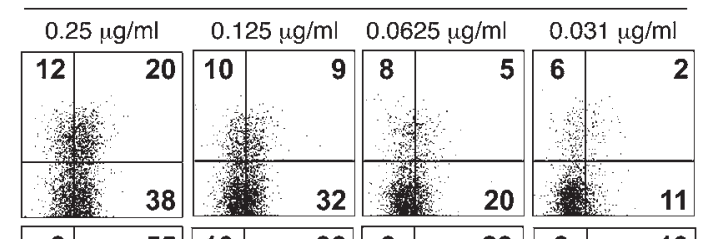

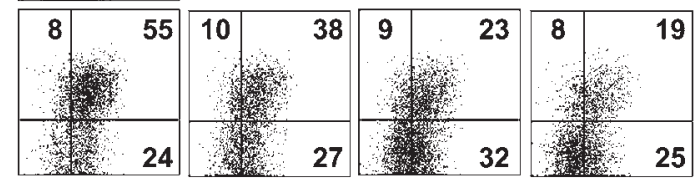

CD25
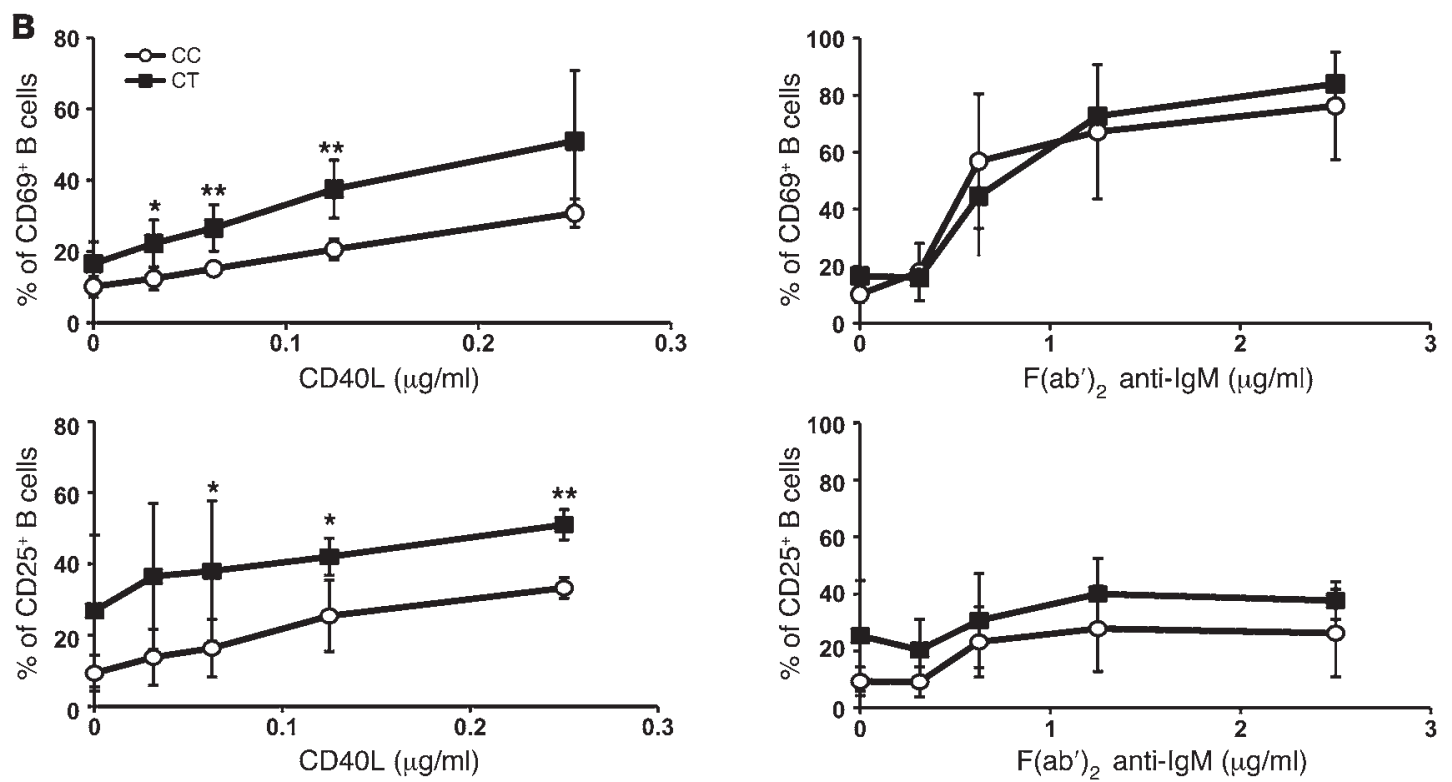

\section{Figure 7}

Enhanced CD40 responses in naive B cells from PTPN22 risk allele carriers. Representative dot plots (A) and combined average expression (B) of CD25 and CD69 on naive B cells from healthy donors carrying or not the PTPN22 risk allele are shown after stimulation in vitro with various indicated concentrations of multimeric soluble recombinant $\mathrm{CD} 40 \mathrm{~L}$ (left) or $\mathrm{F}\left(\mathrm{ab}{ }^{\prime}\right)_{2}$ anti-IgM (right) for 2 days. Values in A represent the percentage of positive cells in each quadrant. Results for HDC10 (non-carrier of the PTPN22 risk allele) and HDP07 (carrier of one PTPN22 risk allele) are shown. Data are representative of 4 independent experiments, and statistical differences are indicated $\left({ }^{\star} P<0.05,{ }^{*} P<0.01\right)$.

beads (Miltenyi). Enriched B cells were stained with FITC anti-human CD27, phycoerythrin (PE) anti-human CD10, anti-human IgM biotin revealed using streptavidin-PECy7, and allophycocyanin (APC) anti-human CD21, all from BD Biosciences - Pharmingen. Single CD2 $1^{\mathrm{lo}} \mathrm{CD} 10^{+} \mathrm{IgM}^{\mathrm{hi}} \mathrm{CD} 27^{-}$ new emigrant/transitional and $\mathrm{CD} 21^{+} \mathrm{CD} 10^{-} \mathrm{IgM}^{+} \mathrm{CD} 27^{-}$peripheral mature naive $B$ cells from patients and control donors were sorted on a FACSVantage (BD) into 96-well PCR plates containing $4 \mu$ lysis solution $(0.5 \times$ PBS containing $10 \mathrm{mM}$ DTT, $8 \mathrm{U}$ RNAsin [Promega], and $0.4 \mathrm{U}$ 5'-3' RNase Inhibitor [Eppendorf]) and immediately frozen on dry ice.

cDNA, RT-PCR, antibody production, and purification. RNA from single cells was reverse transcribed in the original 96 -well plate in $12.5-\mu 1$ reactions containing $100 \mathrm{U}$ Superscript II RT (Invitrogen) for 45 minutes at $42^{\circ} \mathrm{C}$. RT-PCR reactions, primer sequences, cloning strategy, expression vectors, antibody expression, and purification were as described previously $(5,29)$. Immunoglobulin sequences were analyzed by IgBLAST comparison with GenBank. Heavy chain CDR3 was defined as the interval between the conserved arginine/lysine at position 94 in $\mathrm{V}_{\mathrm{H}}$ framework 3 and the conserved tryptophan at position 103 in $\mathrm{J}_{\mathrm{H}}$ segments.
ELISAs and IFAs. Antibody concentrations and reactivity were as described previously $(5,29)$. Highly polyreactive ED38 was used as positive control in HEp-2 reactivity and polyreactivity ELISAs $(5,29)$. Antibodies were considered polyreactive when they recognized all 4 analyzed antigens, which included single-stranded DNA (ssDNA), dsDNA, insulin, and LPS.

For indirect immunofluorescence assays, HEp-2 cell-coated slides (Bion Enterprises Ltd.) were incubated in a moist chamber at room temperature with purified recombinant antibodies at $50-100 \mu \mathrm{g} / \mathrm{ml}$. FITC-conjugated goat anti-human IgG was used as detection reagent.

Microarray gene expression profile analysis. RNA was extracted from $1 \times 10^{5}$ to $3 \times 10^{5}$ batch sorted $\mathrm{CD} 19^{+} \mathrm{CD} 10^{-} \mathrm{CD} 21^{+} \mathrm{CD} 27^{-}$conventional mature naive B cells using the Absolutely RNA Microprep Kit (Stratagene). 100-200 ng of RNA was obtained per sample, and the quality of the purified RNA was assessed by the Bioanalyzer from Agilent Technologies. Using the Ovation biotin system kit from NuGEN, 30-50 ng of RNA was amplified and labeled to produce cDNA. Labeled cDNA was hybridized on chips containing the whole human genome (Human Genome U133 Plus 2.0 Array from Affymetrix). The data discussed in this article have been deposited in the 
National Center for Biotechnology Information's Gene Expression Omnibus and are accessible through GEO series accession number GSE 24736 (http://www.ncbi.nlm.nih.gov/geo/query/acc.cgi?acc=GSE24736).

Real-time quantitative RT-PCR. Mature naive B cells were enriched from the blood of healthy donors by negative selection using the Naive B Cell Isolation Kit II (Miltenyi), followed by the depletion of transitional/new emigrant $B$ cells after PE anti-human CD10 staining and anti-PE magnetic bead treatment (Miltenyi). Total RNA was extracted from mature naive B cells using the Absolutely RNA Microprep Kit, followed by cDNA synthesis with the Superscript II RT. Real-time quantification was performed with an iCycler IQ5 thermal cycler (Bio-Rad) using Evagreen (Bio-Rad) and primers reported in Supplemental Table 53. Actin primers were a gift from O. Henegariu, Yale University. Quantification of the gene of interest was analyzed by the $\Delta \mathrm{C}_{\mathrm{t}}$ method using ACTIN as the reference gene. Relative expression was calculated according the formula $2^{- \text {(CTgene-CTactin). }}$

$B$ cell activation. Naive B cells were plated at 150,000-200,000 cells per well in a 96-well plate in RPMI $10 \%$ serum and various concentrations of polyclonal $\mathrm{F}\left(\mathrm{ab}^{\prime}\right)_{2}$ rabbit anti-human IgM (Jackson ImmunoResearch Laboratories Inc.) or multimeric soluble recombinant human CD40L (Alexis Biochemicals) for 48 hours. Flow cytometry analysis was performed using anti-CD25-FITC, CD86-APC (BioLegend), CD69-PE, CD19-PECy7, CD95/ Fas-APC (BD Biosciences - Pharmingen).

Statistics. Differences were analyzed for statistical significance with 2-tailed unpaired Student's $t$ tests, using SigmaPlot software (Systat). A $P$ value of less than 0.05 was considered significant.

1. Leslie D, Lipsky P, Notkins AL. Autoantibodies as predictors of disease. J Clin Invest. 2001; 108(10):1417-1422.

2. Edwards JCW, et al. Efficacy of B cell-targeted therapy with Rituximab in patients with rheumatoid arthritis. N Engl J Med. 2004;350(25):2572-2581.

3. Hauser SL, et al. B-cell depletion with rituximab in relapsing-remitting multiple sclerosis. $\mathrm{NEnglJ} \mathrm{Med}$. 2008;358(7):676-688.

4. Pescovitz MD, et al. Rituximab, B-lymphocyte depletion, and preservation of beta-cell function. NEngl J Med. 2009;361(22):2143-2152.

5. Wardemann H, Yurasov S, Schaefer A, Young JW, Meffre E, Nussenzweig MC. Predominant autoantibody production by early human B cell precursors. Science. 2003;301(5638):1374-1377.

6. Samuels J, Ng Y-S, Coupillaud C, Paget D, Meffre E. Impaired early B cell tolerance in patients with rheumatoid arthritis. J Exp Med. 2005;201(10):1659-1667.

7. Yurasov S, et al. Defective B cell tolerance checkpoints in systemic lupus erythematosus. J Exp Med. 2005;201(5):703-711.

8. Menard L, Samuels J, Ng YS, Meffre E. Inflammation-independent defective early B cell tolerance checkpoints in rheumatoid arthritis. Arthritis Rheum. 2011;63(5):1237-1245.

9. Gregersen PK, Olsson LM. Recent advances in the genetics of autoimmune disease. Annu Rev Immunol. 2009;27:363-391.

10. Begovich $A B$, et al. A missense single-nucleotide polymorphism in a gene encoding a protein tyrosine phosphatase (PTPN22) is associated with rheumatoid arthritis. Am J Hum Genet. 2004;75(2):330-337.

11. Kyogoku C, et al. Genetic association of the R620W 75(3):504-507.

\section{Acknowledgments}

We thank S. Rudchenko for cell sorting. This work was supported by grants T32 AI089704 (to L. Menard), AI061093, AI071087, and AI082713 from the NIH/National Institute of Allergy and Infectious Diseases and 33-2008-406 from the JDRF (to E. Meffre); by the JDRF-Center for Translational Research (to J.H. Buckner); and by NIH grant DK077905 (to C. Abraham). In addition, we thank the Benaroya Research Institute Translational Research Program and Diabetes Clinical Research Unit for subject recruitment.

Received for publication November 15, 2010, and accepted in revised form June 3, 2011.

Address correspondence to: Eric Meffre, Yale University School of Medicine, 300 George Street, New Haven, Connecticut 06511, USA. Phone: 203.737.4535; Fax: 203.785.7903; E-mail: Eric.meffre@ yale.edu.

David Saadoun's present address is: Department of Internal Medicine and Laboratory I3 "Immunology, Immunopathology, Immunotherapy," UMR CNRS 7211, INSERM U959, Groupe Hospitalier Pitié-Salpetrière, Université Pierre et Marie Curie, Paris, France.

Isabelle Isnardi's present address is: Novartis, Basel, Switzerland. polymorphism of protein tyrosine phosphatase PTPN22 with human SLE. Am J Hum Genet. 2004;

12. Bottini $\mathrm{N}$, et al. A functional variant of lymphoid tyrosine phosphatase is associated with type 1 diabetes. Nat Genet. 2004;36(4):337-338.

13. Michou L, et al. Linkage proof for PTPN22, a rheumatoid arthritis susceptibility gene and a human autoimmunity gene. Proc Natl Acad Sci U S A. 2007; 104(5):1649-1654.

14. Vang T, et al. Autoimmune-associated lymphoid tyrosine phosphatase is a gain-of-function variant. Nat Genet. 2005;37(12):1317-1319.

15. Arechiga AF, et al. Cutting edge: the PTPN22 allelic variant associated with autoimmunity impairs B cell signaling. J Immunol. 2009;182(6):3343-3347.

16. Ng Y-S, Wardemann H, Chelnis J, CunninghamRundles C, Meffre E. Bruton's tyrosine kinase (Btk) is essential for human B cell tolerance. J Exp Med. 2004;200(7):927-934.

17. Tsuiji M, Yurasov S, Velinzon K, Thomas S, Nussenzweig MC, Wardemann H. A checkpoint for autoreactivity in human IgM+ memory B cell development. J Exp Med. 2006;203(2):393-400.

18. Hervé $\mathrm{M}$, et al. CD40 ligand and MHC class II expression are essential for human peripheral $\mathrm{B}$ cell tolerance. J Exp Med. 2007;204(7):1583-1593.

19. Kuroki K, et al. Polymorphisms of human CD19 gene: possible association with susceptibility to systemic lupus erythematosus in Japanese. Genes Immun. 2002;3(suppl 1):S21-S30.

20. Kumar KR, et al. Regulation of B cell tolerance by the lupus susceptibility gene Ly108. Science. 2006; 312(5780):1665-1669.
21. Shlomchik MJ. Sites and stages of autoreactive B cell activation and regulation. Immunity. 2008; 28(1):18-28.

22. Bynoe MS, Grimaldi CM, Diamond B. Estrogen upregulates $\mathrm{Bcl}-2$ and blocks tolerance induction of naive B cells. Proc Natl Acad Sci U S A. 2000;97(6):2703-2708.

23. Graham RR, et al. A common haplotype of interferon regulatory factor 5 (IRF5) regulates splicing and expression and is associated with increased risk of systemic lupus erythematosus. Nat Genet. 2006; 38(5):550-555.

24. Richez C, et al. IFN regulatory factor 5 is required for disease development in the FcgammaRIIB ${ }^{-/-Y a a}$ and FcgammaRIIB-/- mouse models of systemic lupus erythematosus. JImmunol. 2010;184(2):796-806.

25. Azarian M, et al. The PTPN22 R620W polymorphism is associated with severe bacterial infections after human leukocyte antigen geno-identical haematopoietic stem-cell transplantations. Transplantation. 2008;85(12):1859-1862.

26. Lamsyah H, et al. Association of PTPN22 gene functional variants with development of pulmonary tuberculosis in Moroccan population. Tissue Antigens. 2009;74(3):228-232.

27. Haynes BF, et al. Cardiolipin polyspecific autoreactivity in two broadly neutralizing HIV-1 antibodies. Science. 2005;308(5730):1906-1908.

28. Mouquet $\mathrm{H}$, et al. Polyreactivity increases the apparent affinity of anti-HIV antibodies by heteroligation. Nature. 2010;467(7315):591-595.

29. Meffre E, Schaefer A, Wardemann H, Wilson P, Davis E, Nussenzweig MC. Surrogate light chain expressing human peripheral B cells produce self-reactive antibodies. J Exp Med. 2004;199(1):145-150. 